\title{
Radioterapia en tumores móviles
}

\section{Radiotherapy of mobile tumors}

\author{
A. Sola, E. Martínez López, M. Rico, P. Romero, M.T. Vila, E. Villafranca
}

\section{RESUMEN}

En el presente trabajo, se detallan algunas cuestiones relacionadas con el manejo del tratamiento con radioterapia de los tumores móviles, es decir, aquellos que se desplazan con los movimientos respiratorios, integrando el movimiento en el plan de tratamiento. Este hecho complica la administración de dosis altas de radioterapia ya que, en estos casos, el margen de radiación debe ser más amplio de lo que el tumor en sí exige, suponiendo un mayor riesgo para el tejido sano circundante. Sin embargo, las nuevas tecnologías ofrecen una alternativa en estos casos, como son el traking y el gating respiratorio en radioterapia (RT), es decir, la sincronización del tratamiento con el movimiento respiratorio.

En el gating capturamos el tumor y demás órganos de riesgo en un momento determinado del ciclo respiratorio, mientras que en el traking realizamos un "rastreo" del tumor y de los órganos de riesgo a lo largo del ciclo respiratorio, siendo entonces fundamental contar con una buena adquisición de imágenes y una correlación de las mismas con cada fase del ciclo respiratorio.

Los tumores en los que más se han utilizado estas estrategias son los de pulmón, mama y linfomas y con menos frecuencia en algunos abdominales como páncreas, hígado y próstata.

Palabras clave. Tumores móviles. TC-4D. Gating. Traking.

\begin{abstract}
In this article we detail some questions related to managing the treatment of mobile tumors, that is those tumors that shift with respiratory movements, integrating movement into the plan of treatment. This fact complicates the administration of high doses of radiotherapy since, in such cases, the radiation margin must be wider than that required by the tumor itself, representing a greater risk to surrounding healthy tissue. However, the new technologies offer an alternative in these cases, such as tracking and respiratory gating in radiotherapy (RT), that is, the synchronization of treatment with respiratory movement.

In gating we capture the tumor and other organs at risk at a specific moment in the breathing cycle, while in tracking we trace the tumor and the organs at risk throughout the breathing cycle. It is therefore essential to obtain good images and to correlate them with each phase of the breathing cycle.

The tumors with which these strategies have been most employed are those of the lung, breast and lymphomas, and less frequently with some abdominal tumors such as pancreas, liver and prostate.
\end{abstract}

Key words. Mobile tumors. TC-4D. Gating. Tracking.
Servicio de Oncología Radioterápica. Hospital de Navarra. Pamplona.

\author{
Correspondencia \\ Amaya Sola Galarza \\ Servicio de Oncología Radioterápica \\ Hospital de Navarra \\ Irunlarrea, 3 \\ 31008 Pamplona \\ Tfno. 848428475 \\ E-mail: amaia.sola.galarza@navarra.es
}




\section{INTRODUCCIÓN}

Los avances tecnológicos recientes en RT, como la RT de intensidad modulada (IMRT), proporcionan la capacidad de administrar una dosis con distribución homogénea sobre un volumen previamente establecido. Sin embargo, los errores de tratamiento relacionados con la variación de volumen durante el curso del mismo pueden disminuir la eficacia, sobre todo en lesiones situadas en la región torácica y abdominal, especialmente si se administra una única sesión o de forma hipofraccionada, desarrollándose varias técnicas de RT guiada por imagen para manejar esta cuestión. Mediante el gating respiratorio somos capaces de seguir el movimiento del tumor de forma que sólo lo tratamos en una de las fases del ciclo respiratorio, esto nos permite disminuir el volumen de tratamiento y poder administrar dosis mucho más altas que con técnicas convencionales.

En una visión simplificada, el tratamiento con RT se basa en dos pasos fundamentales: la preparación del tratamiento (simulación) y la ejecución del mismo. En la fase de simulación se adquiere mediante TC la información geométrica del paciente y del tumor, las cuales se remiten a un terminal de trabajo que reconstruye un paciente modelo en 3 dimensiones (3D). En la fase de planificación, se diseña el tratamiento sobre el paciente modelo, intentando encontrar un plan de tratamiento adecuado para el paciente específico, preservando los órganos de riesgo. En cada sesión de tratamiento, el paciente se coloca bajo el acelerador lineal, intentando hacer coincidir su geometría con la del paciente modelo de referencia adquirida en el sistema de coordenadas. De esta forma, uno puede ver, para asegurarse de que el tratamiento se realiza tal y como se ha previsto que la geometría del paciente durante todo el tratamiento no cambia. Sin embargo, esto no es tarea fácil, ya que la geometría del paciente, sobre todo la posición y forma del tumor varían de sesión a sesión. Cómo mantener la geometría del paciente próxima al modelo de referencia del sistema de coordenadas de la máquina o cómo poner al día el modelo para acomodar la geometría del paciente, son los temas de investigación de la radioterapia guiada por imagen (IGRT).

La diferencia real geométrica del paciente durante el tratamiento con la del paciente modelo de referencia puede proceder de varias fuentes, tales como el posicionamiento del paciente, la dimensión del órgano, el movimiento respiratorio o el movimiento cardíaco. Además, la dosis de radiación sobre el órgano puede inducir cambios/variaciones en el mismo ${ }^{1}$ (Fig. 1).

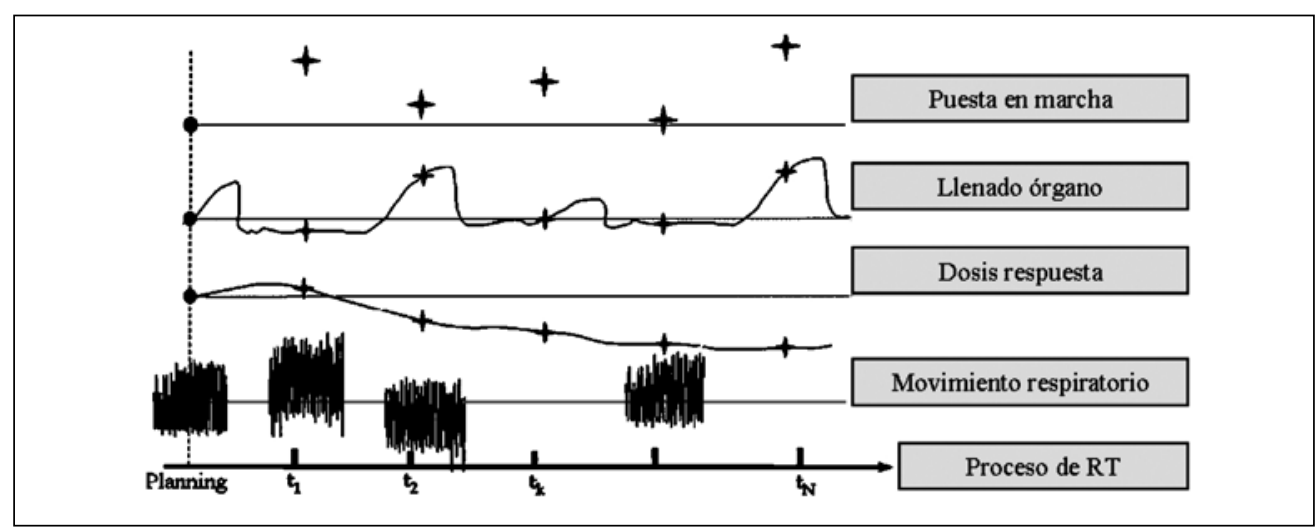

Figura 1. Variación de geometría durante el tratamiento radioterápico. El eje de abscisas representa la fracción de tratamiento y el de ordenadas la posición de tumor ${ }^{1}$. 
Según la escala de tiempo, la variación de geometría se clasifica en variación intrafracción y en interfracción. La variación interfracción ocurre entre las distintas sesiones de tratamiento y se caracteriza por la desviación media de la geometría del paciente en cada fracción de tratamiento sobre la del paciente modelo de referencia; mientras que la intrafracción ocurre durante el transcurso de la irradiación y se debe al cambio inmediato en la geometría del paciente, por lo general causada por el movimiento interno del órgano.

Dado que la mayoría de tipos de movimiento de un órgano contienen una parte inter e intrafracción no muy valorable, la posición de un órgano móvil puede dividirse en posición media y posición inmediata en relación con la posición media. Aquí se introduce el concepto llamado "punto de partida diario de un órgano móvil", que es la posición realizada sobre un promedio de la sesión. Sin embargo, esto puede corresponder a una fase particular de respiración. Por ejemplo, si se realiza un tratamiento con gaiting, la fase al final de la espiración (FFE) define el punto de partida del tumor como la posición FFE realizada en un promedio de la sesión. En la práctica habitual, el punto de partida de un órgano móvil en una sesión de tratamiento se mide al inicio del mismo y puede variar después del inicio.

\section{FASE DE SIMULACIÓN EN EL TRATAMIENTO DE TUMORES MÓVILES}

Tal y como se ha mencionado antes, el objetivo de la TC de simulación es obtener toda la información geométrica del paciente y construir un paciente modelo de referencia en el ordenador para proceder a la planificación del tratamiento. El paciente modelo de referencia contiene todos los órganos de riesgo así como el volumen tumoral a tratar. Los errores en la delimitación del volumen tumoral a tratar influirán en todas las sesiones de tratamiento por lo que deben de manejarse con cuidado. Se ha discutido mucho si la influencia de la velocidad de exploración, el tipo de TC (helicoidal, multicorte, en abanico), el número cortes (cortes finos versus extremadamente groseros), mala calibración de la TC de simulación tenía algo que ver con este tipo de errores.

En relación a la velocidad de movimiento del tumor los artefactos pueden aparecen de diversas formas, así:

- Si la velocidad de la TC de exploración es más lenta que la velocidad de movimiento del tumor obtenemos una imagen distorsionada del mismo. Esto ocurría antiguamente, aunque actualmente algunos especialistas reducen la velocidad de exploración con la intención de conseguir una imagen distorsionada del tumor para crear un volumen tumoral interno. De todas maneras no se recomienda esta técnica ya que es difícil de interpretar el número de cortes distorsionados en el TC, dificultando así la delimitación del volumen tumoral. Esto es similar a la exploración con tomografía por emisión de positrones (PET) que tarda en realizarse unos veinte minutos $y$ genera una imagen cuyo volumen es difícil de interpretar.

- Si la velocidad de la TC de exploración es más rápida que la velocidad de movimiento del tumor obtendríamos una imagen del tumor en una fase arbitraria de la respiración. Esto se produciría en caso de realizar múltiples cortes en TC o "cone-beam TC".

- Si la velocidad de exploración es similar a la velocidad de movimiento del tumor, como es el caso de las TC helicoidales actuales, la posición y forma del tumor se deforma. Este hecho ha sido estudiado por Chen y $\mathrm{col}^{2}$, basándose en un estudio dónde concluyeron que las distorsiones a lo largo del eje de movimiento pueden causar sobreestimación del volumen a considerar (Fig. 2). 


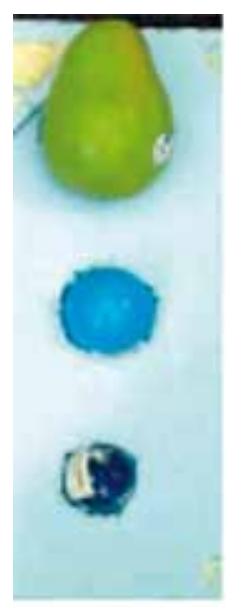

(a)
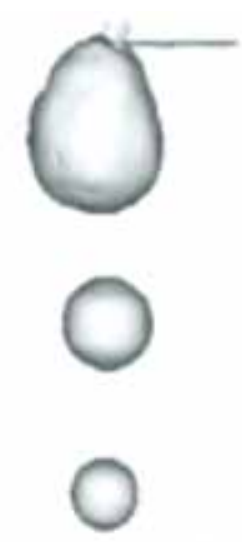

(b)
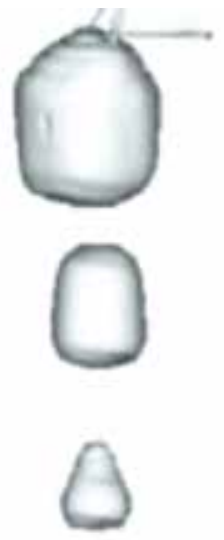

(c)
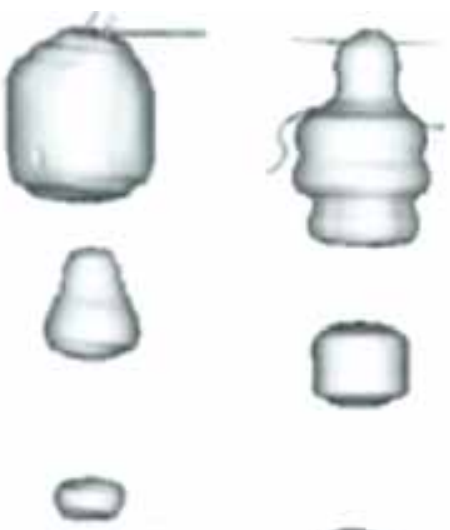

(d)

\section{(e)}

Figura 2. Artefactos que produce el movimiento en la TC de simulación. A y B representan objetos estáticos y C, D y E en movimiento².

Este último punto es el más relevante en la práctica clínica. Una forma de minimizar los artefactos de movimiento consiste en que los pacientes aprendan a controlar la respiración mientras reciben la sesión de tratamiento, sin embargo para muchas de ellos esto es muy dificultoso, sobre todo para los que tienen una función pulmonar deficiente. La respuesta actual a este problema es la utilización de la información anatómica del paciente referenciada a los cambios temporales producidos por el ciclo respiratorio, es decir, a lo que comúnmente se denomina "imagen $4 \mathrm{D}$ ", administrando de manera continua el tratamiento y adaptándonos en cada momento a la posición del tumor y a la deformación de la anatomía debida a la respiración.

En una revisión reciente realizada por $\mathrm{Keall}^{3}$, se destacan dos aspectos importantes. El primero es que la ventaja de la TC $4 \mathrm{D}$, respecto a la TC $3 \mathrm{D}$, es que no sólo se reducen los errores de movimiento, sino que además la información acerca del movimiento del órgano y del tumor queda codificada en un conjunto de imágenes en cuatro dimensiones. El segundo es que la TC $4 \mathrm{D}$ no es realmente en cuatro dimensiones, sino que la información temporal es un ciclo respiratorio a lo largo del cual se distribuyen las imágenes de la TC 3D. Las variaciones en la amplitud entre los ciclos respiratorios causan artefactos en las imágenes de TC 4D. Una solución a este problema es entrenar al paciente con la respiración y así de esta manera, asegurar un modelo de respiración regular durante todo el proceso de simulación ${ }^{4}$.

\section{TÉCNICAS DE TRATAMIENTO EN LOS TUMORES MÓVILES}

El modelo tradicional para cubrir el movimiento del órgano, consiste en añadir un margen de seguridad al volumen blanco clínico (CTV) consiguiendo de esta forma la planificación del volumen a tratar. Este margen de seguridad debe de realizarse con cuidado y tiene que ser lo suficientemente grande para asegurar una dosis total adecuada sobre el órgano móvil y lo suficientemente pequeño para preservar los tejidos sanos adyacentes. El objetivo principal de la IGRT es reducir el margen CTVPTV (volumen blanco de planificación), de modo que puedan reducirse las complicaciones sobre el tejido sano. 
La mayor parte del margen se utiliza para minimizar el movimiento inter e intrafracción. Mediante la implantación en el paciente de marcadores fiduciales y el seguimiento de los mismos con radiografías diarias, teóricamente se puede reducir el movimiento interfracción a un nivel significativo. El tamaño del margen se determina según la información que tenemos acerca del movimiento del tumor de un paciente específico, de las técnicas utilizadas para la simulación de tratamiento y de la puesta en marcha del mismo. La magnitud del movimiento intrafracción puede estimarse mediante la media de la población tratada y la información medida para un paciente específico durante la simulación de tratamiento o el TC 4D. En la mayoría de los casos el margen de un paciente concreto es menor que el de la media de la población. La forma de controlar el movimiento intrafracción es construir el volumen intencionado a tratar (ITV) basado en una exploración de TC $4 \mathrm{D}^{5}$. Debe tenerse especial cuidado y usar la información de movimiento específica para cada paciente ya que tal información se mide en el momento de la simulación de tratamiento y no puede representarse con exactitud dada la variación geométrica del paciente. Por tanto, debería de supervisarse el movimiento del tumor durante el tratamiento con técnicas de dirección de imagen o asegurando un modelo de respiración regular, entrenando al paciente.

Los métodos para integrar el movimiento del órgano al tratamiento pueden dividirse en dos categorías:

1. Controlar el movimiento del tumor mediante el mantenimiento de la respiración o la compresión abdominal.

2. Permitir el libre movimiento del tumor durante el ciclo respiratorio.

Los métodos para el control del movimiento del tumor, a menudo, requieren una participación activa del paciente y del oncólogo radioterápico que participa en dicho tratamiento ${ }^{6-9}$. Este método no suele tolerarse bien por aquellos pacientes con una función pulmonar deficiente, hecho que ocurre en la mayoría de los pacientes con cáncer de pulmón. El gating respiratorio limita la irradiación a la parte del ciclo respiratorio en el que el tumor se encuentra en el camino con el haz de tratamiento. En su inicio fue puesto en marcha mediante un sistema robótico de radiocirugía (Ciberknife). Para el acelerador lineal, el movimiento del tumor puede compensarse mediante un colimador multiláminas. Entre las técnicas anteriormente descritas para manejar el movimiento del tumor, el gating repiratorio se considera exacto y fácil de poner en marcha.

\section{GATING EN RADIOTERAPIA}

El tratamiento con gating respiratorio pretende reducir la incidencia y la severidad de las complicaciones en el tejido sano y aumentar el control local mediante una escalada de dosis administrada en tumores móviles localizados en tórax y abdomen. La localización exacta del tumor en tiempo real es importante para el gating debido al margen reducido CTV-PTV y/o a la intensificación de dosis. Hay que diferenciar el gating del tracking; mientras que en el gating capturamos el tumor y demás órganos en un momento determinado del ciclo respiratorio obteniendo imágenes en un momento determinado de ese ciclo, en el tracking capturamos el tumor y demás órganos de riesgo a lo largo del ciclo respiratorio obteniendo imágenes en distintas fases de la respiración que se interpolan y a las que se les da una orden temporal en consonancia con la fase respiratoria que les corresponde.

La localización directa del tumor en tiempo real es difícil, pero no es imposible. Existen dos formas de gating en radioterapia. El gating interno, que utiliza el movimiento del tumor interno mediante marcadores fiduciales implantados en el paciente, y el gating externo que utiliza los movimientos respiratorios externos, como por ejemplo, marcadores colocados sobre la superficie abdominal del paciente.

El único sistema de gating interno usado en la práctica clínica es el que rastrea el tumor en tiempo real (RTRT), desarrollado por la compañía Mitsibishi, Ltd, Tokio, Japón en colaboración con la Universidad de Hokkaido ${ }^{10-12}$. La dirección de posición en tiempo real (revoluciones por minuto), 
desarrollado por Sistema Varian, es la representación del gating externo.

El sistema desarrollado por la compañía electrónica Mitsubishi, consta de 4 cámaras de rayos X; en cada una de las cuales existe un tubo de rayos $\mathrm{X}$ colocado sobre el suelo y con un intensificador de imagen de 9 pulgadas $(22,86 \mathrm{~cm})$ situado en el techo con un generador de rayos $\mathrm{X}$ de alto voltaje.

Los 4 tubos de rayos X están dispuestos, dos en posición craneal (derecha de izquierda) y otros dos en posición caudal (derecha e izquierda) con respecto al paciente y a una distancia de $280 \mathrm{~cm}$ del isocentro. El intensificador de imagen está situado en el techo, enfrente de los tubos de rayos $\mathrm{X}$ y a una distancia de $180 \mathrm{~cm}$ del isocentro y con el eje central cruzando dicho isocentro. En un tiempo dado durante el tratamiento, según el ángulo del cabezal del acelerador, dos de los 4 sistemas de rayos $\mathrm{X}$ adquieren dos imágenes que son registradas por dos sistemas de imagen portal. Para reducir la radiación dispersa del haz, las unidades de rayos $\mathrm{X}$ y el acelerador se sincronizan (el haz de Megavoltaje se para cuando se pulsan las unidades de Kilovoltaje) (Fig. 3).

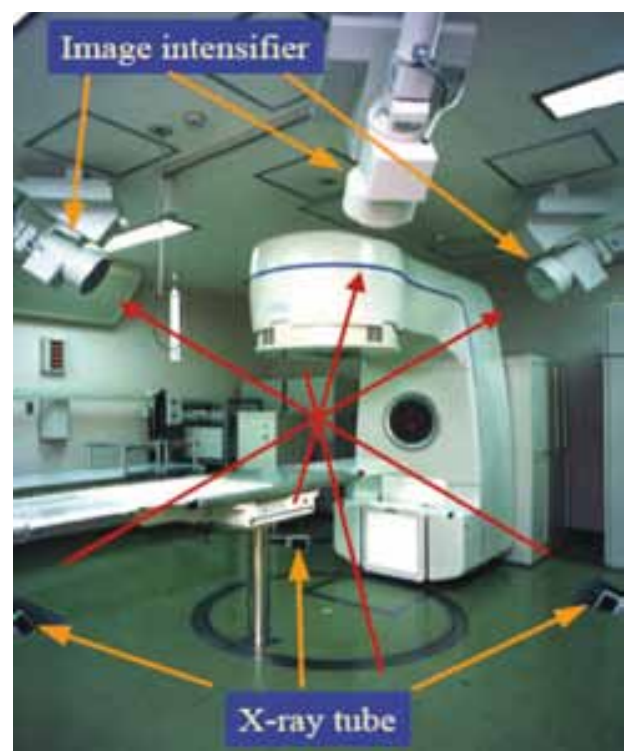

Figura 3. Sistema desarrollado por Mitsubishi Electronics Co, Ltd, Tokio, Japan que rastrea el tumor en tiempo real (RTRT) en colaboración con la Universidad de Hokkaido ${ }^{10-12}$.
El sistema de revoluciones por minuto se ha puesto en práctica y se ha investigado en un amplio número de centros ${ }^{13-17}$. Un bloque ligero de plástico marcado con 2 marcadores pasivos reflexivos infrarrojos, se coloca sobre la superficie abdominal del paciente y se supervisa por una cámara de vídeo. La señal es el movimiento abdominal superficial que realiza el paciente. Un filtro periódico comprueba la forma regular de la curva de respiración, e inmediatamente inutiliza el haz cuando la forma de la curva de respiración se hace irregular, como por ejemplo al moverse el paciente o cuando tose, permitiendo de nuevo al haz establecerse cuando la respiración se hace de nuevo regular (Fig. 4).

Las fuentes principales de gating externo no son invasivas y son relativamente fáciles, no requiriendo ninguna dosis de irradiación adicional para obtener la imagen, sin embargo, no son equivalentes al rastreo del tumor y pueden causar errores. En particular, la relación entre el movimiento de tumor y la señal realizada pueden cambiar el tiempo levemente.

Cuando usamos este sistema, los marcadores fiduciales implantados en el tumor pueden rastrearse mediante fluoroscopía ${ }^{18}$. El acelerador lineal sólo irradia el tumor cuando el marcador está dentro de la ventana interna del gating. El tamaño de la ventana es de 1-3 mm de acuerdo con las características del paciente y el margen utilizado en la planificación de tratamiento ${ }^{11}$. Las técnicas para insertar los marcadores fiduciales (que poseen un diámetro de 1,5-2 $\mathrm{mm}$ ) en el tumor o cerca de su superficie, incluyen desde la broncoscopia en el caso de un tumor en la periferia del pulmón, inserción percutánea en caso de metástasis hepáticas, cistoscópica percutánea para la inserción en la próstata y quirúrgica para lesiones espinales y paraespinales ${ }^{12,19}$.

La implantación de marcadores fiduciales es un procedimiento invasivo, que permite visualizar la posición del tejido blando para determinados movimientos internos del órgano, teniendo esto múltiples riesgos potenciales. Al obtener imágenes en tiempo real se puede elegir si adquirirlas antes, 
después o incluso durante el tratamiento, de esta forma podemos verificar no sólo movimientos interfracción sino también intrafracción. Muchos oncólogos radioterápicos tienen poca predisposición para utilizar estos procedimientos en pacientes con cáncer de pulmón debido al alto riesgo de neumotórax. La inserción de marcadores con el broncoscopio es factible y segura, pero sólo para ciertas lesiones pulmonares ${ }^{19-21}$.

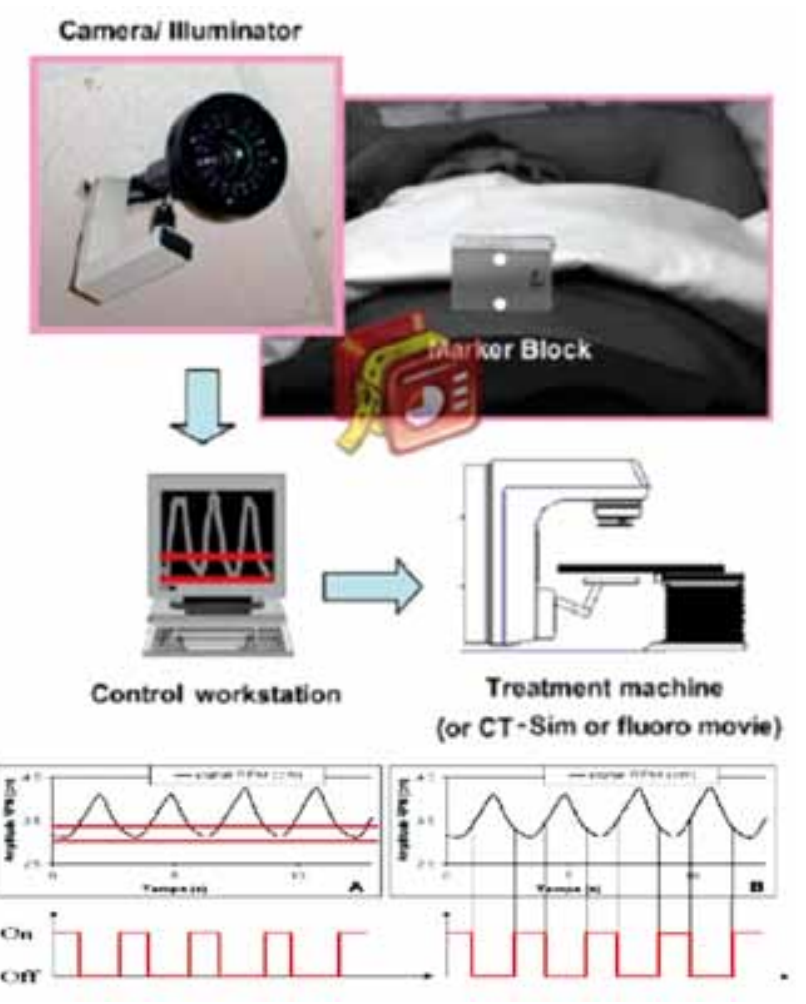

Figura 4. En esta figura observamos el marcador externo colocado sobre la superficie abdominal del paciente, el cual refleja una señal luminosa que se detecta por la cámara enviando una señal al ordenador, el cual la procesa e inutiliza el haz de irradiación cuando esta se hace irregular ${ }^{13-17}$.
La dosis estimada para la imagen portal en el tratamiento con RTRT es de especial interés. Esta imagen portal se adquiere a través de unos dispositivos electrónicos que se encuentran integrados en la propia unidad de tratamiento. Tienen su inconveniente en que el proceso de lectura de la imagen está sincronizado con pulsos de radiación. La dosis estimada en RTRT puede aumentar aproximadamente a 1,2 mGy/ $\mathrm{h}^{22}$, dato que hay que tener en consideración.

Los marcadores internos implantados son buenos sustitutos en el posicionamiento del tumor y no hay publicadas posibles migraciones del marcador si las imágenes de simulación se adquieren un día después de la implantación del marcador ${ }^{19,23}$. Esto preocupa aún menos si se implantan múltiples marcadores. Los dos problemas principales del gating interno son el riesgo de neumotórax por implantación de marcadores en el pulmón y la alta dosis de imagen requerida para el rastreo fluoroscópico. Por lo tanto, el gating interno es exacto, pero "caro" o poco práctico según la localización del tumor.

\section{PROBLEMAS Y SOLUCCIONES}

El rechazo para la puesta en marcha del gating externo se refleja por una falta de confianza en la posición del tumor que se obtie- 
ne a través de marcadores externos. Si esta confianza se pudiera establecer mediante una correlación interna-externa, el gating externo podría ser aceptado por la mayoría de los oncólogos radioterápicos. En la práctica habitual, el gating interno se basa en la implantación de marcadores fiduciarios, sin embargo, debido al alto riesgo de neumotórax, la inserción de estos marcadores en el pulmón no es un procedimiento que se realice habitualmente. Sin estos marcadores, la tecnología actual no nos permite rastrear tumores pulmonares en el gating interno.

Otro problema de especial interés son las altas dosis que se requieren para el rastreo fluoroscópico en el gating interno, por lo que éstas deben reducirse considerablemente, antes de que el gating interno sea un procedimiento aceptado en la práctica clínica habitual.

Para la realización del gating externo, se captura una señal respiratoria externa "S". La ventana externa del gating corresponde a un segmento de señal externa con la que se conecta el haz de tratamiento. Por lo general, la ventana externa del gating se escoge al final de la espiración, ya que esta fase tiene una duración más larga y es más fácil de reproducir. Para simplificar el tema, se reduce la ventana gating a un punto, "S0", es decir, sólo conectamos el haz cuando la S=S0.

Se han utilizado diversos métodos para grabar el ciclo respiratorio. Uno de ellos, comercializado por VARIAN consiste en colocar sobre el paciente un pequeño maniquí con unos puntos o marcas reflectantes de luz infrarroja, que se mueve simultáneamente con la respiración. Mientras se realiza la TC de planificación, con un sistema de vídeo se graba el movimiento de los puntos, que se traduce en una curva representativa del ciclo respiratorio. Con un software especial se analiza la curva y se clasifican y ordenan los cortes de TC en relación con su posición temporal en la curva de la respiración. De esta forma se dispone de varios conjuntos de cortes de $\mathrm{TC}$, cada uno de los cuales corresponde a una fase del ciclo respiratorio. El volumen total se funde en un TC-4D en la fase final de la espiración, que se usa como TC de planificación. Los órganos de riesgo se contornean sobre el TC al final de la espiración y se añade un margen al CTV para obtener el PTV, desarrollando así un plan de tratamiento con radioterapia conformada 3D (RTC3D) o con IMRT.

Debido al movimiento interfracción, el punto de partida del tumor en relación con los tatuajes de la piel o de referencias óseas puede variar considerablemente de fracción a fracción.

Una forma de reducir al mínimo esta variación es entrenando la respiración del paciente, obteniendo así un modelo de respiración más estable y regular. Esta técnica también se llevó a cabo en el MGH, expresamente para la amplitud gating en la fase final de la espiración. El sistema gating de revoluciones por minuto tiene una función de regeneración visual que permite al paciente observar la forma de curva de respiración en tiempo real. Dos líneas consecutivas que contienen posiciones al final de la espiración se usan para definir la amplitud. Al observar el paciente su propia curva de respiración mediante un par de gafas protectoras, se le pide en la fase final de la espiración mantener la respiración entre esas dos líneas. Este procedimiento se aplica en cada paso del tratamiento, desde la simulación, a la fase de comprobación, a la administración de tratamiento, asegurando una posición estable al final de la espiración (Figs. 5 y 6 ).

Incluso con el entrenamiento del paciente, son necesarios sistemas de verificación del tratamiento para asegurarse que los puntos de partida del tumor están dentro del haz de tratamiento.

Otra técnica de verificación en el tratamiento con RTC3D y gating respiratorio se desarrolló en el MGH utilizando marcadores fiduciales. La posición de estos marcadores se detectan a través de la emisión y recepción de ondas de radiofrecuencia por una matriz de emisores/receptores electromagnéticos colocados encima de la superficie del paciente (sin contacto con éste). A su vez, la posición de la matriz de receptores relativa al sistema de coordenadas de la sala de tratamiento se determina a través 
de unos sensores de infrarrojo colocados en la matriz que se comunican con tres cámaras colocadas en el techo de la sala de tratamiento y que están calibradas respecto al isocentro (punto en torno al cual gira el origen de la irradiación) del acelerador lineal. La combinación de la posición de la matriz de receptores en la sala de tratamiento y la posición de los marcadores respecto a la matriz nos proporciona la posición de los marcadores relativa al isocentro. Estas posiciones de los marcadores se transmiten a una consola en la que se muestran de forma continua las variaciones en la posición longitudinal, lateral y vertical de los marcadores respecto a su posición inicial en función del tiempo verificando que estas variaciones se mantienen dentro de unas tolerancias establecidas. Las posiciones se actualizan a una frecuencia de $10 \mathrm{~Hz}$, lo que permite un seguimiento prácticamente continuo de la posición del volumen durante el tratamiento. Aunque el sistema, que se utiliza básicamente para tratamientos de próstata, permite la monitorización continua de la posición de los marcadores, no genera imágenes anatómicas del volumen. De todas formas, el uso de radiaciones no ionizantes permite el ahorro de dosis al paciente durante el proceso de posicionamiento y localización respecto a otros sistemas de imagen.
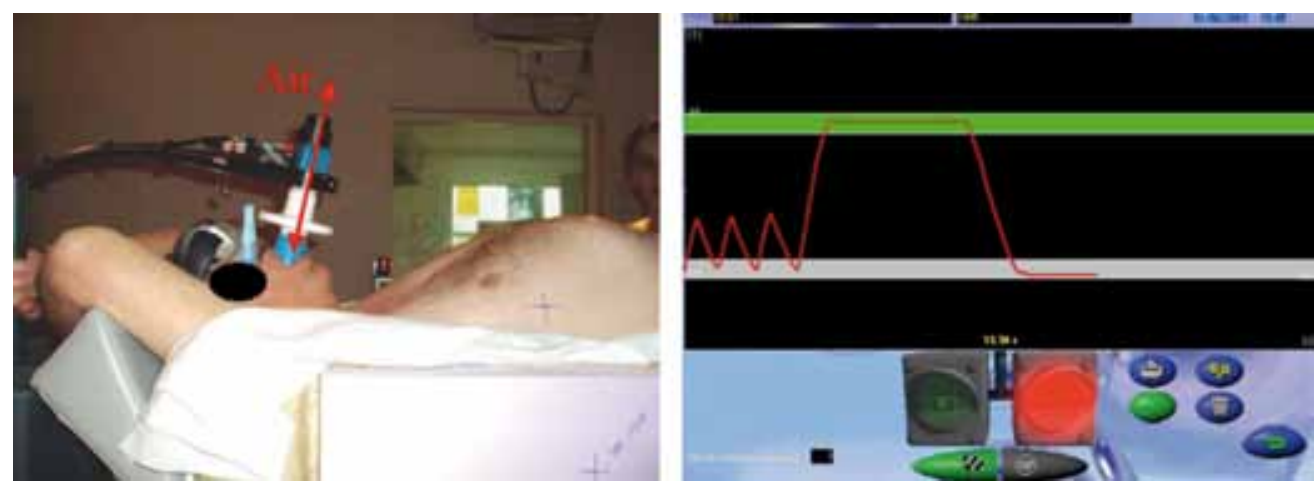

Figura 5. El paciente observa su propia curva de respiración en tiempo real mediante unas gafas protectoras, pudiendo controlar su propia respiración. En este caso se realiza una inspiración forzada tratando el tumor en dicho momento.

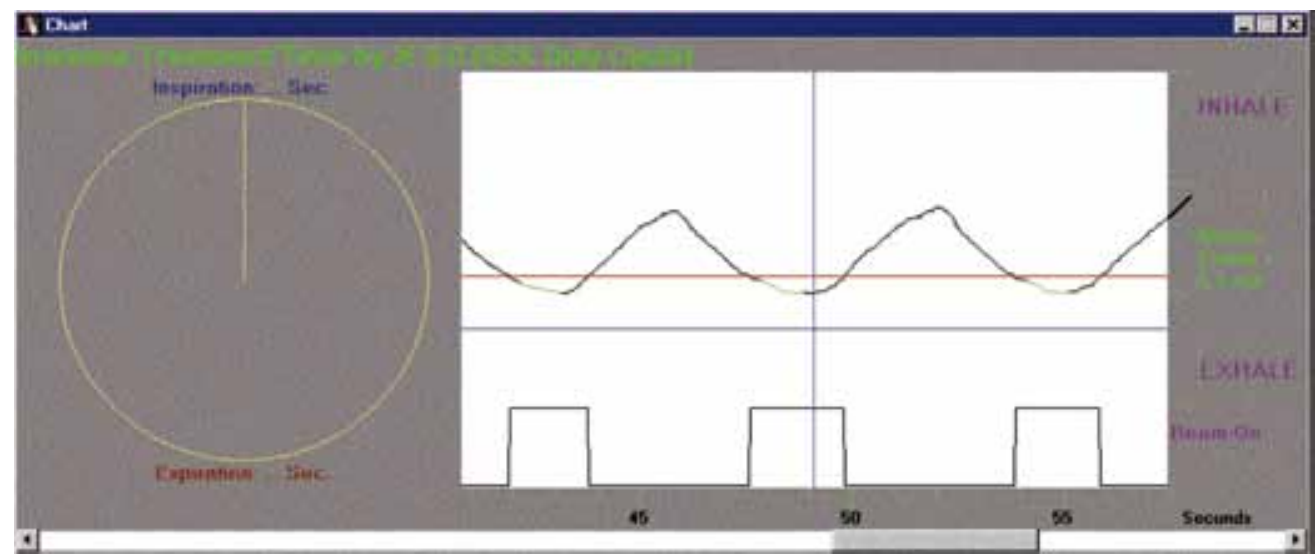

Figura 6. Representación del ciclo respiratorio en función del tiempo (eje de abcisas) y de la amplitud del movimiento (eje ordenadas). En la fase de inspiración la curva se dirige hacia arriba y en la espiración hacia abajo. La parte inferior de la figura representa el momento en el que se administra la radiación. 


\section{BIBLIOGRAFÍA}

1. Yan D, Lockman D, Martinez A, Wong J, BrabBINS D, VICINI F et al. Computed tomography guided management of interfractional patient variation. Semin Radiat Oncol 2005; 15 : 168-179.

2. Chen GT, Kung JH, Beaudette KP. Artifacts in computed tomography scanning of moving objects. Semin Radiat Oncol 2004; 14: 19-26.

3. Keall P. 4 Dimensional computed tomography imaging and treatment planning. Semen Radiat Oncol 2004; 14: 81-90.

4. Neicu T, Berbeco R, Wolfgang J. Jiang SB. Synchronized moving apertura radiation therapy (SMART): Improvement of breathing pattern reproducibility using respiratory coaching. Phys Med Biol 2006; 51: 617-636.

5. Rietzel E, Chen G, Choi N, Willet C. Tour-dimensional image-based treatment planning: Target volume segmentation and dose calculation in the presence of respiratory motion. Int J Radiat Oncol Biol Phys 2005; 61: 1535-1550.

6. Mah D, Hanley J, Rosenzweig KE, Yorke E, BraBAN L, LING CC et al. Technical aspects of the deep inspiration breath-hold technique in the treatment of thoracic cancer. Int J Radiat Oncol Biol Phys 2000; 48: 1175-1185.

7. Rosenzweig Ke, Hanley J, Mah D, Mageras G, Hunt M, Toner S et al. The deep inspiration breath-hold technique in the treatment of inoperable non-small-cell lung cancer. Int $\mathrm{J}$ Radiat Oncol Biol Phys 2000; 48: 81-87.

8. Remouchamps VM, Letts N, Vicini FA, Sharpe MB, Kestin LL, Chen PY et al. Initial clinical experience with moderate deep-inspiration breath hold using an active breathing control device in the treatment of patients with left-sided breast cancer using external beam radiation therapy. Int $\mathrm{J}$ Radiat Oncol Biol Phys 2003; 56: 704-715.

9. Remouchamps VM, Letts N, Yan D, Vicini FA, Moreau M, Zielinski JA et al. Three-dimensional evaluation of intra- and interfraction immobilization of lung and chest wall using active breathing control: a reproducibility study with breast cancer patients. Int J Radiat Oncol Biol Phys 2003; 57: 968-978.

10. Shimizu S, Shirato H, Kitamura K, Shinohara N, HaRabayashi T, Tsukamoto $\mathrm{T}$ et al. Use of an implanted marker and real-time tracking of the marker for the positioning of prostate and bladder cancers. Int J Radiat Oncol Biol Phys 2000; 48: 1591-1597.

11. Shimizu S, Shirato H, Ogura S, Akita-Dosaka H, Kitamura K, Nishioka T et al. Detection of lung tumor movement in real-time tumor-tracking radiotherapy. Int $\mathrm{J}$ Radiat Oncol Biol Phys 2001; 51: 304-310.

12. Shirato H, Shimizu S, Kitamura K, Nishioka T, Kagei K, Hashimoto $S$ et al. Four-dimensional treatment planning and fluoroscopic real-time tumor tracking radiotherapy for moving tumor. Int J Radiat Oncol Biol Phys 2000; 48: 435-442.

13. Vedam SS, Kini VR, Keall PJ, Ramakrishnan V, Mostafavi H, Mohan R. Quantifying the predictability of diaphragm motion during respiration with a noninvasive external marker. Med Phys 2003; 30: 505-513.

14. Kini VR, Vedam SS, Keall PJ, Patil S, Chen C, Монал R. Patient training in respiratory-gated radiotherapy. Med Dosim 2003; 28: 7-11.

15. Mageras GS, Yorke E. Deep inspiration breath hold and respiratory gating strategies for reducing organ motion in radiation treatment. Semin Radiat Oncol 2004; 14: 6575.

16. Mageras GS, Yorke E, Rosenzweig K, Braban L, Keatley E, Ford E et al. Fluoroscopic evaluation of diaphragmatic motion reduction with a respiratory gated radiotherapy system. J Appl Clin Med Phys 2001; 2: 191-200.

17. Ford EC, Mageras GS, Yorke E, Rosenzweig KE, WAGMAN R, Ling CC. Evaluation of respiratory movement during gated radiotherapy using film and electronic portal imaging. Int J Radiat Oncol Biol Phys 2002; 52: 522-531.

18. Shirato H, Shimizu S, Kunieda T, Kitamura K, Van Herk M, Kagei $\mathrm{K}$ et al. Physical aspects of a real-time tumor-tracking system for gated radiotherapy. Int J Radiat Oncol Biol Phys 2000; 48: 1187-1195.

19. Harada T, Shirato H, Ogura S, Oizumi, Yamazaki $\mathrm{K}$, Shimizu $\mathrm{S}$ et al. Real-time tumor-tracking radiation therapy for lung carcinoma by the aid of insertion of a gold marker using bronchofiberscopy. Cancer 2002; 95: 1720-1727.

20. Shirato H, Harada T, Harabayashi T, Hida K, Endo H, Kitamura $\mathrm{K}$ et al. Feasibility of insertion/ implantation of 2.0-mm-diameter gold internal fiducial markers for precise setup and real-time tumor tracking in radiotherapy. Int J Radiat Oncol Biol Phys 2003; 56: 240-247. 
21. Imura M, Yamazaki K, Shirato H, Onimaru R, FuJINo M, SHIMIzU $\mathrm{S}$ et al. Insertion and fixation of fiducial markers for setup and tracking of lung tumors in radiotherapy. Int J Radiat Oncol Biol Phys 2005 63: 1442-1447.

22. Shirato H, Oita M, Fujita K, Watanabe Y, Miyasaka K. Feasibility of synchronization of real-time tumor-tracking radiotherapy and intensitymodulated radiotherapy from viewpoint of excessive dose from fluoroscopy. Int J Radiat Oncol Biol Phys 2004; 60: 335-341.

23. Kitamura K, Shirato H, Shimizu S, Shinohara N, Harabayashi T, Shimizu T et al. Registration accuracy and possible migration of internal fiducial gold marker implanted in pros- tate and liver treated with real-time tumor-tracking radiation therapy (RTRT). Radiother Oncol 2002; 62: 275-281. 
